\title{
Concrete Continuum: \\ Concept, Calculus, \& Construction Connected Through Parametric Representation
}

\author{
Tristan Al-Haddad \\ Georgia Institute of Technology, USA \\ tristan@gatech.edu \\ Keyan Rahimzadeh \\ Georgia Institute of Technology, USA \\ keyanr@gmail.com
}

Jill Fredrickson

Georgia Institute of Technology, USA

jillfred890@gmail.com

\begin{abstract}
This paper outlines a custom-built suite of scripts that automate the processes of reinforced concrete design and is directly linked to the parametric design model of the architect. The workflow creates a design and engineering feedback loop for early phase schematic design. Using this system, the design geometry is generated and then deconstructed into a Finite Element model. The workflow executes a static analysis then calculates rebar size and placement, and finally generates fabrication drawings. This methodology allows architectural intent and engineering analysis to be collapsed into a single non-linear design process.
\end{abstract}

Keywords: Parametric design; Digital fabrication; Reinforced concrete; Production automation; Design feedback process.

\section{Introduction}

The following is both a case study and methodological outline for linking the various activities of any design and construction project in a non-linear process of shared parametric representations between various participants. This linkage allows the team to marry the conceptual and the constructive domains via computational tools. The implications of connecting these domains through automation are myriad. Perhaps the most significant is the instant feedback provided to the designer while making early schematic design decisions. In particular, a specific configuration of structural elements can be informed by feedback of structural requirements and material implications for fabrication. For example, while manipulating a column's angle of tilt, the designer is immediately provided with descriptions of resulting rebar configurations, material takeoffs, constructability, and cost. By providing active feedback into the design process, architects are empowered to intuitively understand the physical implications of their aesthetic intentions (Al-Haddad, 2009).

This research was conducted as a two-semester graduate design studio at the Georgia Institute of Technology in 2012-2013. The course was a design/build studio with the challenge of creating a small public performance space on the Atlanta Beltline. The Beltline is a transformational infrastructure project that, when complete, will convert 22 miles of underutilized rail corridors into a looped public park encircling the city center (beltline.org). The charge of the studio was to design, engineer, and construct the structure using a reinforced concrete superstructure to create an intervention in the landscape and support a custom Photovoltaic lighting feature, intended to further define this intimate space for the arts.

\section{Workflow}

The overall workflow consists of a series of stages defined by a conversion of information from one format to another, such as geometric to analytical, or 2-d to $3-d$. The initial design process consisted of an internal competition within the studio that created the overall framework and design intent from which the initial design model resulted. This paper focuses on the transformation of that model into multiple representations of critical information. The initial design model is converted into a structural analysis model seeded by the design geometry and some guidance vectors. The results of this analysis drive a rebar design routine, producing 2 - $d$ diagrams of rebar arrangements which are then expanded into a $3 \mathrm{~d}$ model. That model, in combination with the original design model, then provides all of the geometric information required for automating the fabrication documentation. These results may at any point compel the designer to alter the design iteration and restart the process, creating a feedback loop between the design and the engineering processes.

\section{Design Intent}

The abandoned rail corridor site, scattered with rusting rails from the 1800's and bearing the topographic scars of cut and fill grading operations, strongly influenced the studio's design response. Studies of historical rail profiles drove both the formal intent and informed the parametric definition of the design model. The intent was to manipulate the scale and form of these profiles so that they might interact with the human body and create seating and structural elements (Figure 1). 


\section{Design model definition}

To aid in the design process, a script was developed that parametrically represented the geometry of a historical rail with rule-based associative logic and flexible input parameters that defined the major geometric elements (Figure 2). Measurements labeled $\mathrm{A}-\mathrm{H}$ denote adjustable input parameters, which can be modified during design, affecting the 3-dimensional geometry and providing the designer with immediate visual feedback. The diagram below shows the variability that can be achieved by manipulating the input parameters, such as section height, seat width, wall thickness, curvature radius, rotation and scaling. (Figure 3)

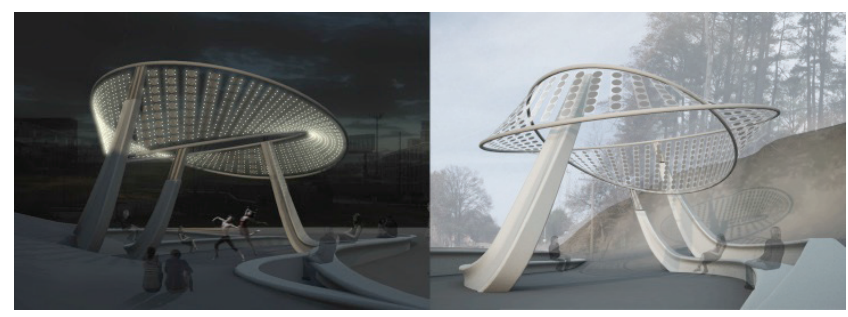

Figure 1: Renderings of project on the Atlanta Beltline.

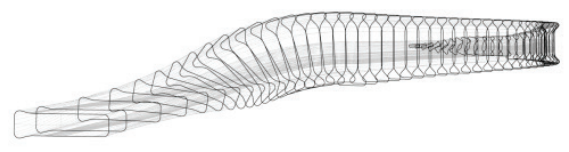

Figure 2: Parametric rail profile and variables
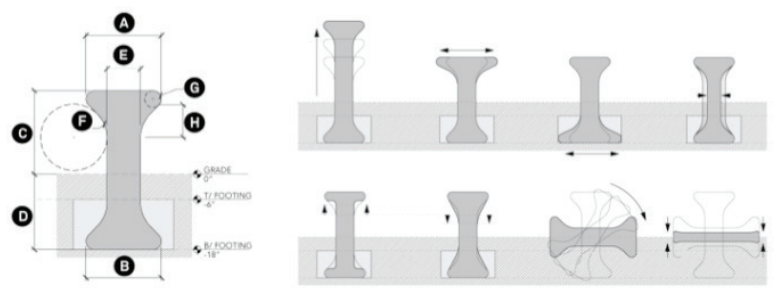

Figure 3: Parametric rail profile and variables

In addition to a flexible sectional rail profile definition, the 3dimensional geometric volumes were intended to be flexible as well. Thus, parametric associations were created such that the profiles would smoothly transform the geometry from path condition to seat to structural column and the designer would control the rate and locations of these transitions. This rule-based, parameter-driven design tool was tremendously useful for quickly generating highly adaptive reinforced concrete infrastructure and allowed alternate schematic and formal ideas to be quickly tested for visual and performative effect along with constructability.
To achieve smooth transitions in geometry, the definition was designed to interpolate the parameters, driving section profile geometry, between locations of design input. The designer could adjust these parameters at the chosen input locations, along userdefined 3-dimensional design curves, and control the rate of transition between these profiles by manipulating control points on bell-curve-like graphs. These profile curves were then lofted in straight sections to produce a segmented geometry of ruled surfaces that appear curved in plan. The interval of these segments was important because it both informed the resolution, or overall smoothness of the geometry, and informed the module of the formwork. After weighing the effects of different resolutions on the scale of the geometry and its rate of curvature/twisting, a 2-foot module was chosen. Additionally this modular segmented geometry was also useful as an input for structural analysis as well as the reinforcing design, however for these purposes the design geometry was often re-sliced at a finer interval. Figure 4 below diagrams the overall design workflow.

\section{Structural Analysis}

The structurally expressive qualities of the design motivated the integration of engineering considerations early in the design process. A set of scripts were developed that would directly link the design model with a structural analysis model, providing immediate feedback of the structural behavior of a particular design iteration.

\section{Analysis model definition}

The first stage in the analytical process was to design a process that would interactively translate design geometry into analytical elements. The process is slightly different for each of the structural systems, of which there are four: 1) the large, cantilever columns, 2) the benches, which perform as low retaining walls, 3) the footings, and 4) the lightweight photovoltaic canopy above (Figure 1). For the benches and the column, the first challenge is tackling the complex geometry of its non-uniform cross-section. Using the geometric tools available in the Rhinoceros plugin Grasshopper (rhino3d.com), the complex profiles were approximated by best-fit rectangular profiles at regular intervals. The $3 d$-NURBS curve that defines the axis of the bench is then segmented into discrete linear sections at the sampling points. Correlating the rectangular approximations with these segments defines the initial table of 1-d Finite Elements. The bench elements are distinct from the columns in that they are connected to the footings, which are represented by a grid of $2 \mathrm{~d}$ plates generated at the base nodes of the bench. The soil spring boundary conditions are assigned their stiffness by the tributary areas of these plates, thus leveraging the geometric tools of the modeling environment to drive some of the tedious calculations, which, if done manually, would be even further complicated by irregular geometry. 
(1) 3d Input Curves Are Subdivided

(2) Planes Generated

At Benches: Planes are vertical At Columns: Planes are normal to curve

(3) Parametric Description Of Rail Propagated To Planes

The input parameters are adjusted at key profile locations
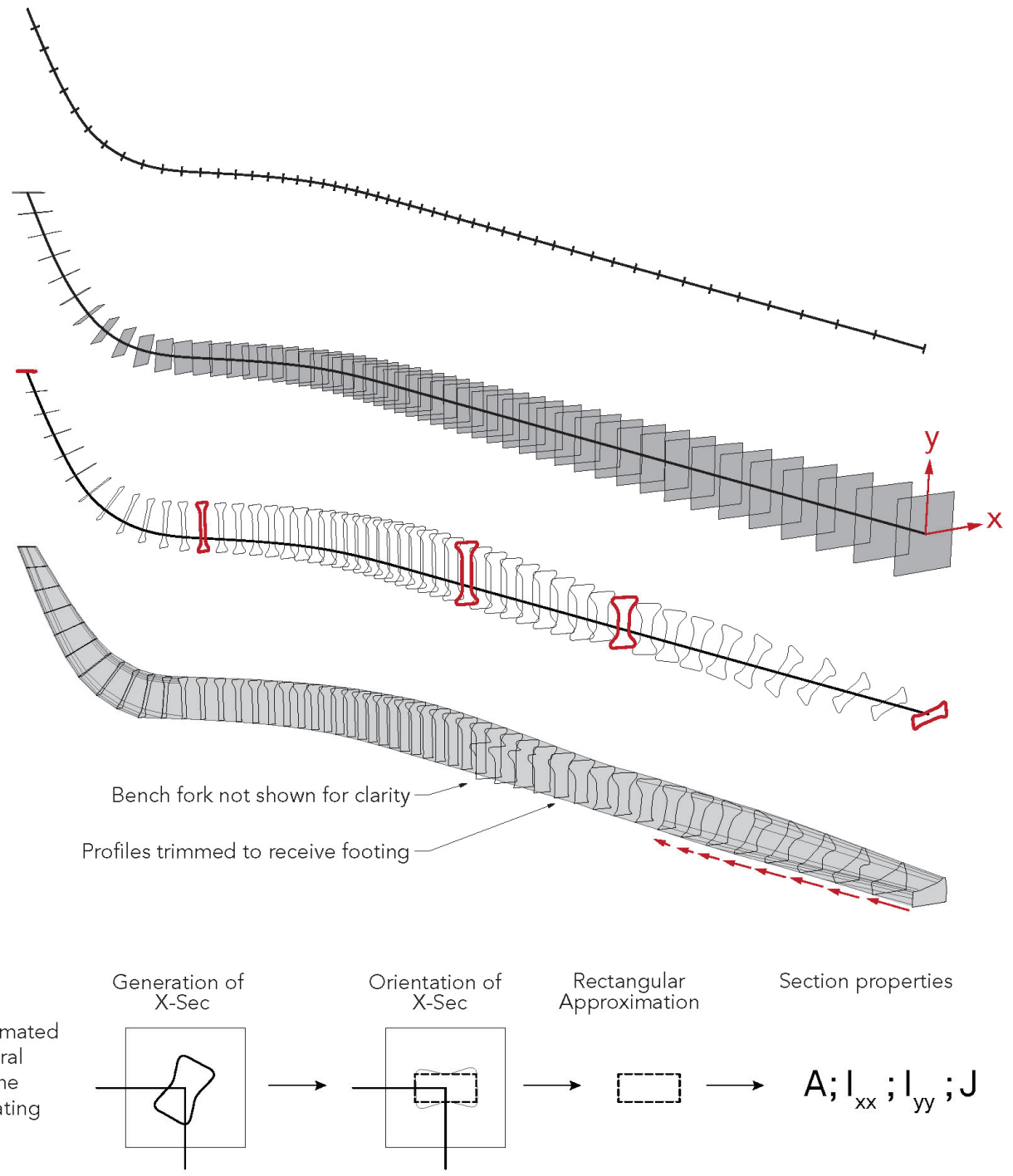

\section{Automated Rebar Design Drawings}

Geometry is analyzed by rebar-generating script at 12-inch intervals (Referencing $\mathrm{ACl} 318$ and the competed structural analysis). $2 \mathrm{~d}$ shop drawings are then output.
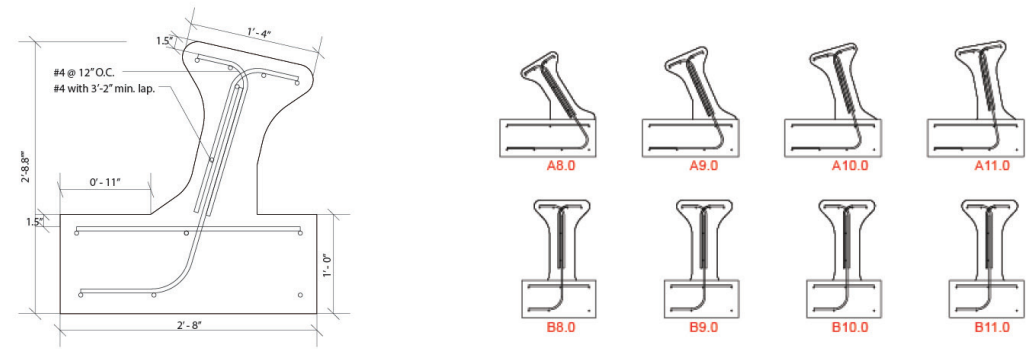

Geometry is parametrically approximated into rectangular sections for structura design and inform the rebar-generating script.
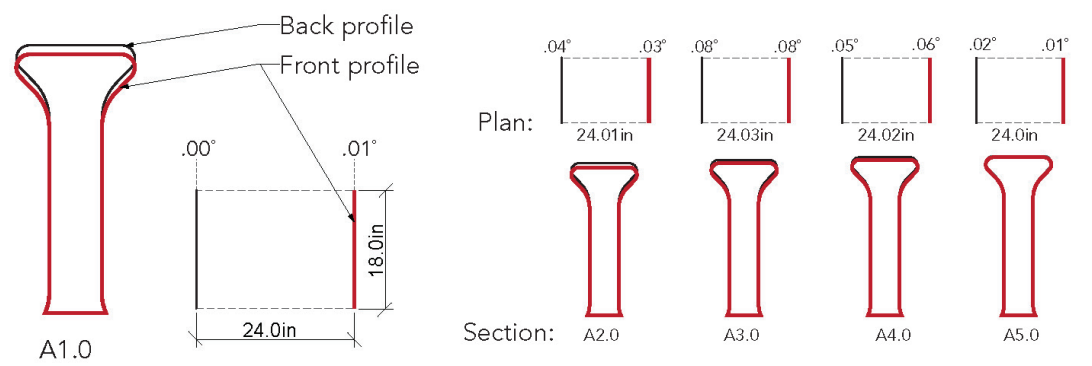

Figure 4: Parametric workflow from design geometry to structural analysis, detailing and fabrication drawings. 
The canopy surfaces were converted to $2 \mathrm{~d}$ Load Patch elements, which do not contribute to structural simulation but only transfer load into the primary structure. The canopy is a lightweight, flexible structural system that is highly subject to nonlinear effects, and requires an independent structural treatment, one possible extension of the work herein.

While these steps are likely familiar to many who have performed a computational structure analysis, the value of this workflow is its instantaneous update; that is, as the design model changes, the engineering model is automatically recompiled, cross-sections updated, tributary areas recalculated, thus reducing the time, effort, and energy spent making incremental design changes.

\section{Analysis}

Structural analysis is executed with all of the Load Cases and Combinations in accordance with ASCE 7. For each structural element, the results for each case are directly assigned to the geometry in the model, such that each segment can be directly queried from within the modeling environment.

\section{Design}

The results are parsed to determine the worst loading condition for each individual element, which is then pushed to a beam design sub-routine that executes a preliminary rebar design. This sub-routine is a first-principle calculation of required steel area based on ultimate moment, moment of inertia, and code-specified coverage requirements. The emphasis is not on producing thoroughly vetted, immediately code compliant results, but to give the designer a first glance at the approximate implications for a design choice, and to directly incorporate the realities of structural behavior into the design process.

The link between analysis results and design geometry enables the translation of rebar calculations into a $3 \mathrm{~d}$ model. With the rebar modeled in its intended place within the matrix of concrete, the $3 \mathrm{~d}$ model can be used to produce the hundreds of rebar drawings that are required for construction. Given that the freeform geometry calls for unique drawings at every location, the automation of this process is a great advantage. It also provides insight for the designer with regard to what construction scenarios he or she is creating, such as areas of high steel congestion, or problematic formwork configurations. With the entire construction model established, fabrication take-offs can be compiled, parts numbered, and fabrication tickets created.

\section{Fabrication}

Upon finalization of the design, the fabrication of formwork is also a parametric, digitally controlled process. The formwork interior mold surface was to be fabricated of expanded polystyrene (EPS) using a computer-controlled 6-axis hot-wire cutter. This would be achieved by sending the two flat profile curves that define a given geometry segment to the machine, along with their spatial offset and relative angles of rotation. The hot-wire would essentially re- loft the geometry as the wire interpolates between front and back profile curves. Secondary cuts would then be made to create wedge-shaped mold segments at locations of extreme curvature. These fabrication drawings were incorporated into the parametric design definition, such that the drawings required for hot-wire fabrication would automatically update following modifications to the overall design. In this manner, complex ruled-surface formwork could be quickly fabricated in segments, and then assembled, adhered, sealed and finished in preparation for casting on site.

\section{Conclusion}

The availability of lightweight tools enabled and accelerated a technically advanced design process that incorporated technical rigor into the demanding timelines of a design build process. As an architectural studio, the emphasis was not to develop a new method of structural analysis, nor was it to develop a totally bespoke analysis environment. In fact, the intent was to use the standard practices and procedures of the construction industry and engineering in particular. In doing so, the results are easily verifiable by hand and in accordance with traditional engineering practice. This enables the engineer to use the outputs of this process as a basis of design, or to engage the workflow at any stage and expand on the results.

Indeed, this capacity for expansion is one of the most compelling results of this investigation. The piecemeal, lightweight construction of these parametric tools is such that they can be continually refined and expanded to meet the needs of the design team. The various experts on the team can engage the workflow at any relevant stage and quickly incorporate deeper levels of sophistication. The most apparent developments to be pursued are the expansion of the beam design processes, and incorporating the specifications in the building code directly into the model, bringing the analysis results closer to verifiable documents. The analysis of the large mat footing that supports the cantilevering column may require specific analysis more advanced than would be appropriate for this workflow, but that is a point that requires further inquiry.

The ultimate realizations of the motivations described above would be an automated optimization routine, wherein a range of input parameters are given along with certain fitness parameters such as total weight, cost of material, construction time. The entire workflow could then be iterated through in pursuit of the most optimal configuration, while ultimately guided by the intent of the designer.

\section{Acknowledgements}

This research was performed with generous support of the Concrete Reinforcing Steel Institute. For analysis, the studio utilized different packages throughout the development of the project, having used Karamba $3 \mathrm{~d}$ as well as Oasys GSA, which was enabled by Jon Mirtschin's Geometry Gym components. 
This project was developed collaboratively under the instruction of Professor Tristan Al-Haddad, with graduate students Dylan Bussey, Graham Cannady, Olivia Hallquist, Jennifer Lewis, Jessica Marquardt, Derrick Tittle, Emily Tuttle, and Erin West, in addition to the authors.

\section{References}

Al-Haddad, T. (2009). Force + Feedback + Fabrication: Integrating Structural Feedback into Design Processes for Complex SurfaceActive Form. Saarbrucken: VDM Verlag.

American Society of Civil Engineers. (2006) Minimum Design Loads for Buildings and Other Structures. Standards ASCE/SEI 7-10. Annapolis Junction, Maryland.

Beltline.org. "Project History."

<http://beltline.org/progress/progress/project-history/> (accessed

September 30, 2013) 